\title{
Primary meningeal melanoma with cerebrospinal fluid dissemination mimicking neurofibromatosis type 2
}

Melanoma meníngeo primário com disseminação liquórica mimetizando neurofibromatose tipo 2

\author{
Marcos Rosa Júnior ${ }^{1}$, Luciene Lage da Motta², Fabrizio Scardino
}

A 38-year-old man admitted with headache, left paresis and bilateral sensorineural hearing loss. Neuroimaging showed a peripheral frontal tumor with hyperintensity on T1WI and bilateral internal auditory canal (IAC) lesions. (Figures 1, 2 and 3). The presence of hyperintensity on T1WI, without fat or hemorrhage

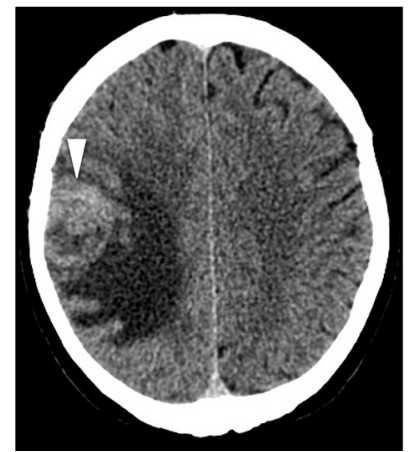

Figure 1. Non-contrast CT showed a right frontal hyperdense tumor (arrowhead).
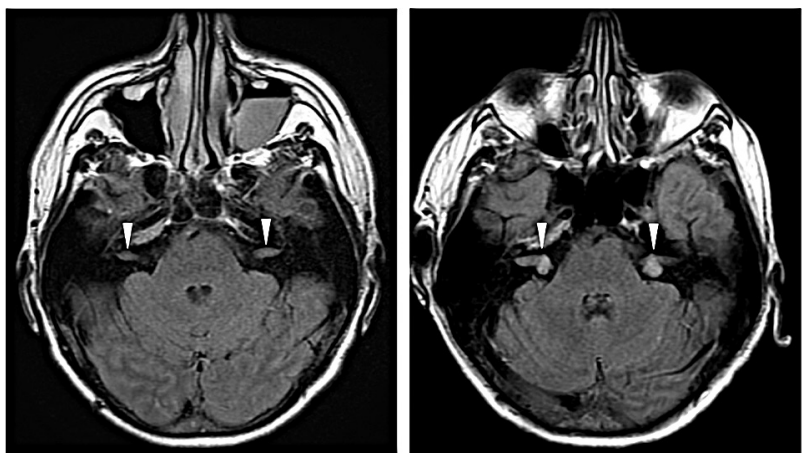

Figure 3. FLAIR showed bilateral IAC lesions (arrowheads) (A), which increased over the following 30 days (arrowheads) (B) mimicking bilateral acoustic schwannoma in neurofibromatosis type 2 . should direct for lesions containing melanin ${ }^{1}$. Resection of the frontal tumor diagnosed a primary malignant meningeal melanoma with cerebrospinal fluid dissemination once the patient has no melanocytic lesions outside the CNS. The melanocytic lesions ranges from melanocytoma to melanoma ${ }^{23,4,5}$.Malignant melanoma should be included in the differential diagnosis of neoplastic CSF dissemination with bilateral IAC lesions mimicking schwannomas in NF2.
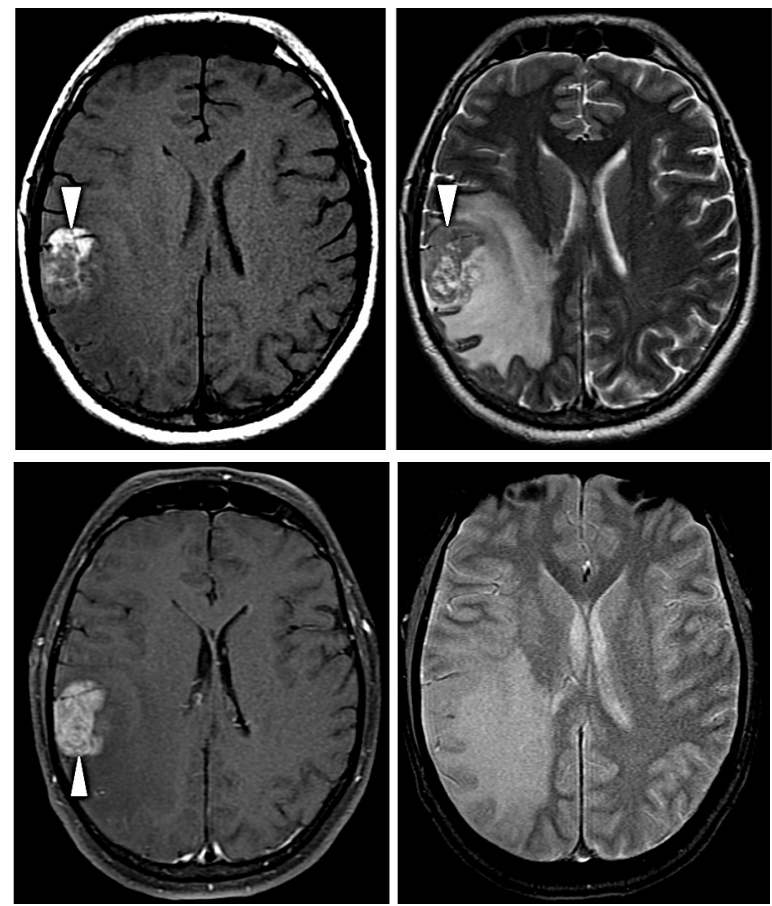

Figure 2. MRI showed a frontal peripheral tumor with signal hyperintensity on T1WI (arrowhead) (A), signal hypointensity on T2WI (arrowhead) (B) and enhancement after contrast administration (arrowhead) (C), without hemorrhage on T2 gradient-echo (D).

\footnotetext{
${ }^{1}$ Universidade Federal do Espírito Santo, Seção de Radiologia, Vitoria ES, Brazil; ${ }^{2}$ Laboratório de Cito e Histopatologia Virchow, Vitoria ES, Brazil;

${ }^{3}$ Hospital Estadual Jayme Santos Neves, Seção de Neurocirurgia, Serra ES, Brazil.

Correspondence: Marcos Rosa Júnior; Centro de Ciências da Saúde, UFES; Avenida Marechal Campos, 1468; 29043-900 Vitória ES, Brasil;

E-mail:marcos.rosa@ufes.br

Conflict of interest: There is no conflict of interest to declare.

Received 26 December 2014; Received in final form 19 February 2015; Accepted 13 March 2015.
} 


\section{References}

1. Ginat DT, Meyers SP. Intracranial lesions with high signal intensity on T1-weighted MR images: differential diagnosis. Radiographics. 2012;32(2):499-516. http://dx.doi.org/10.1148/rg.322105761

2. Brat DJ, Giannini C, Scheithauer BW, Burger PC. Primary melanocytic neoplasms of the central nervous systems. Am J Surg Pathol. 1999;23(7):745-54.

3. Roser F, Nakamura M, Brandis A, Hans V, Vorkapic P, Samii M. Transition from meningeal melanocytoma to primary cerebral melanoma: case report. J Neurosurg. 2004;101(3):528-31. http://dx.doi.org/10.3171/jns.2004.101.3.0528

4. Bydon A, Gutierrez JA, Mahmood A. Meningeal melanocytoma: an aggressive course for a benign tumor. J Neurooncol. 2003;64(3):259-63. http://dx.doi.org/10.1023/A:1025628802228

5. Xie ZY, Hsieh KLC, Tsang YM, Cheung WK, Hsieh CH. Primary leptomeningeal melanoma. J Clin Neurosci. 2014;21(6):1051-2. http://dx.doi.org/10.1016/j.jocn.2013.08.018 